\title{
Changes in Cellular Crosstalk between Skeletal Muscle Myoblasts and Bone Osteoblasts with Aging
}

\author{
Jonathan A. Doering ${ }^{1}$, Carly E. Britt ${ }^{1}$, Gregory S. Sawicki ${ }^{2}$, Jacqueline H. Cole ${ }^{1 *}$ \\ ${ }^{1}$ Joint Department of Biomedical Engineering, University of North Carolina, Chapel Hill, NC, \\ and North Carolina State University, Raleigh, NC, USA \\ ${ }^{2}$ George W. Woodruff School of Mechanical Engineering and School of Biological Sciences, \\ Georgia Institute of Technology, Atlanta, GA, USA
}

\section{*Corresponding author:}

Dr. Jacqueline H. Cole, PhD

Assistant Professor

North Carolina State University \& University of North Carolina-Chapel Hill

4212B Engineering Building III

911 Oval Drive

Campus Box 7115

Raleigh, NC 27695-7115

Telephone: 919-515-5955

Email: jacquecole@ncsu.edu 


\section{Abstract}

2 Musculoskeletal function declines with aging, resulting in an increased incidence of trips and falls.

3 Both bone and muscle experience age-related losses in tissue mass that alter their mechanical

4 interactions in a well characterized manner, but changes in the biochemical interactions between

5 bone and muscle with aging are not well understood. Of note, insulin-like growth factor 1 (IGF-

6 1), a potent growth factor for bone and muscle, can be negatively altered with aging and may help

7 explain losses in these tissues. We recently developed a co-culture system for simultaneous growth

8 of bone mesenchymal stem cells (MSCs) and muscle satellite cells (SCs) to investigate the

9 biochemical crosstalk between the two cell types. Here, we utilized an aging rat model to study

10 cellular changes between young and old rat MSCs and SCs, in particular whether 1) young MSCs

11 and SCs have increased proliferation and differentiation compared to old MSCs and SCs; 2) young

12 cells have increased IGF-1 and collagen expression as a measure of crosstalk compared to old

13 cells; and 3) young cells can mitigate the aging phenotype of old cells in co-culture. Rat MSCs

14 and SCs were either mono- or co-cultured in Transwell ${ }^{\circledR}$ plates, grown to confluence, and allowed

15 to differentiate for 14 days. Across the 14 days, cell proliferation was measured, with

16 differentiation and crosstalk measurements evaluated at 14 days. The results suggest that in both

17 young and old, proliferation is greater in mono-cultures compared to co-cultures, yet age and cell

18 type did not have a significant effect. Differentiation did not differ between young and old cells,

19 yet MSCs and SCs demonstrated the greatest amount of differentiation in co-culture. Finally, age,

20 cell type, and culture type did not have a significant effect on collagen or IGF-1 expression. These

21 results suggest co-culture may have a controlling effect, with the two cell types acting together to

22 promote differentiation more than in mono-cultures, yet this response was not altered by age. In

23 general, results for old cells had higher variability, suggesting a wider variety in the aging 
24 phenotypes demonstrated in these animals. This study was the first to use this rat aging model to

25 investigate changes between bone and skeletal muscle cells, however further investigations are

26 required to determine what signaling changes occur in response to age. Determining these

27 signaling changes could lead to new targets for mitigating the progression of aging.

Introduction

Musculoskeletal performance declines with aging, resulting in an increased incidence of trips

31 and falls in the elderly. ${ }^{1}$ These changes are compounded by concomitant losses in bone mass

32 (osteopenia, osteoporosis) and skeletal muscle mass (sarcopenia), leading to high rates of fracture

33 and muscle injury in the elderly population. ${ }^{3,4}$ Under the mechanostat paradigm, these detrimental

34 muscle changes, which manifest in decreased muscle loading on the skeleton, will induce negative

35 bone adaptation and bone loss. ${ }^{2}$ Aged individuals with decreased bone mass (osteopenia) often

36 also present with reduced muscle mass and function (sarcopenia). ${ }^{3,4}$ However, although elderly

37 populations often experience both osteopenia and sarcopenia, age-related declines in either bone

38 or muscle mass cannot fully explain the extent of functional declines in the other tissue, supporting

39 the idea that bone-muscle interactions are not solely mechanical in nature. Indeed, the emergent

40 thought is that biochemical interactions also play an important role in bone and muscle tissue

41 health and function, in particular the direct communication between the two through paracrine

42 signaling pathways, or cell-cell crosstalk. ${ }^{5,6}$ Improving our understanding of how bone-muscle

43 crosstalk changes with aging could inform the development of targeted therapies to mitigate age-

44 related losses in musculoskeletal function.

45 Age-related changes in biochemical signaling are well characterized for bone and muscle

46 individually. In muscle, satellite cells (SCs) responsible for muscle repair have decreased activity 
47 from overuse, and signaling molecules such as inflammatory and growth factors are reduced. ${ }^{7,8}$

48 Similarly, bone loss associated with aging is the result of reduced functional ability of bone

49 osteoblasts to produce new bone, as well as a loss of signaling factors. ${ }^{9}$

50 While these signaling changes have been investigated in either muscle or bone alone, the

51 investigation of the signaling changes in tandem have been under characterized. The concurrent

52 age-related changes in bone and muscle signaling are not fully understood, although the

53 importance of this crosstalk has been established in several key studies. In elegant parabiosis

54 studies, in which the vascular systems for young and old rat pairs were surgically merged, old rats

55 experienced some muscle rejuvenation, including increased muscle mass and improved muscle

56 fiber structure, although specific factors from the young rats stimulating this change remained

57 unidentified. ${ }^{17,18}$ Further, in vivo mouse studies demonstrated faster healing of bone fractures in

58 the presence of additional xenografted muscle tissue, ${ }^{19,20}$ but again the specific crosstalk factors

59 stimulating tissue repair were ill-defined, where specific signaling factors were not discussed.

60 Therefore, a more specific method is required to investigate the specific crosstalk changes. Our

61 previous work optimized a co-culture system between SCs and bone mesenchymal stem cells

62 (MSCs) to investigate the optimal growth conditions for each cell type. Utilization of this system

63 could lead to better understanding of the crosstalk between muscle and bone.

64 One signaling target for investigation in both muscle and bone is insulin-like growth factor

651 (IGF-1). IGF-1 is a potent growth factor secreted by the liver in response to the secretion of

66 growth hormone, resulting in increased growth of tissues like bone and muscle. ${ }^{10}$ However, IGF-

671 has also been demonstrated to be released by both muscle and bone to be used in an autocrine or

68 paracrine fashion. ${ }^{10,11}$ Further, reduction in IGF-1 lead to reductions in both muscle and bone

69 mass, ${ }^{11-13}$ and decreased the amount of collagen in these tissues. ${ }^{14-16}$ However, how IGF-1 is 
70 altered in the crosstalk between muscle and bone has not been investigated.

71 The purpose of this study was to characterize concurrent changes in bone and muscle cells

72 and biochemical interactions with aging. Using our previously optimized co-culture system, we

73 paired bone mesenchymal stem cells (MSCs) and muscle SCs from both young and old rats and

74 examined cell viability, differentiation, and crosstalk using young-young, old-old, young-old, and

75 old-young pairs. We hypothesized that in old cells, there would be decreased cell viability,

76 differentiation, and crosstalk compared to young cells. Furthermore, we wanted to test the

77 hypothesis that young factors could improve the aging phenotype as demonstrated by Conboy et

78 al. ${ }^{18}$ By co-culturing young and old cells together we hypothesized that the young SCs or MSCs

79 when cultured with the opposite old cell would be unchanged when compared to their regular

80 young co-cultured with young cells, however, old cells under the influence of young cells would

81 have increased cell viability, cell differentiation, and cell crosstalk when compared to old cells co-

82 cultured with other old cells.

$84 \quad$ Methods

85 Animals and cell isolation

86 The Institutional Animal Care and Use Committee at North Carolina State University 87 approved all animal procedures. F344 x Brown Norway F1 hybrid (F344BN) rats were used in this 88 study as a model for aging, because they are commonly used in aging muscle function studies. Ten 89 young (5-9 months old) and ten old (33-34 months old) F344BN rats (National Institutes of Aging,

90 Bethesda, Maryland) were fed a normal diet and kept under a 12-hr light/12-hr dark cycle with

91 free access to chow and water until euthanasia. After acclimation to the animal facility for one 92 week, rats were euthanized by $\mathrm{CO}_{2}$ inhalation, followed by immediate dissection to remove the 
93 gastrocnemius and tibialis anterior (TA) muscles and the tibia and femur from one leg. Rat bone

94 MSCs were isolated from the tibia and femur using a method modified from Zhang and Chan. ${ }^{21}$

95 Briefly, the bones were placed in warm MSC-specific growth medium (MSCGM) consisting of

96 alpha-MEM (Gibco, Gaithersburg, MD), 10\% fetal bovine serum (Gemini Bio-Products, West

97 Sacramento, CA), and $100 \mathrm{U} / \mathrm{mL}$ penicillin-100 $\mu \mathrm{g} / \mathrm{mL}$ streptomycin (Gibco). The bone ends were

98 removed, and the remaining segments were flushed with MSCGM to extract the marrow and

99 isolate the MSCs. The MSCs were plated on a single 120-mm plastic plate (Corning, Tewksbury,

100 MA) coated with 2\% gelatin (Fisher Scientific, Hampton, NH) and cultured with MSCGM in a

101 humidified atmosphere at $37^{\circ} \mathrm{C}$ with $5 \% \mathrm{CO}_{2}$. After $48 \mathrm{~h}$, the nonadherent cells were washed out,

102 and the cultures were expanded to passage 2.

103 SCs were extracted from the gastrocnemius and TA using a protocol from Danoviz and

104 Yablonk-Reuveni. ${ }^{22}$ Briefly, the muscles were placed in warm SC-specific growth medium

105 (SCGM) consisting of high glucose Dulbecco’s MEM (Gibco), 20\% fetal bovine serum (Gemini

106 Bioproducts), 10\% horse serum (Gibco), and $100 \mathrm{U} / \mathrm{mL}$ penicillin-100 $\mu \mathrm{g} / \mathrm{mL}$ streptomycin

107 (Gibco). Tendon, fat, blood vessels, and connective tissue were removed, and the remaining

108 muscle was cut into small $\left(\sim 3 \mathrm{~mm}^{3}\right)$ cubes. Muscle cubes were collected and added to a $1 \%$ Pronase

109 solution (Pronase Protease, Millipore-Sigma, St. Louis, MO) in SCGM for one hour for digestion.

110 The solution and remaining muscle were then collected and suspended in $10 \mathrm{~mL}$ of additional

111 SCGM. The entire solution was subjected to mechanical trituration by passing the solution through

112 a wide-bore pipette to liberate SCs. Finally, the triturated solution was passed through a cell

113 strainer with a 70- $\mu \mathrm{m}$ pore size (Fisher Scientific), and SCs were collected and plated on a single

$1142 \%$ gelatin-coated $120-\mathrm{mm}$ plate with SCGM in a humidified atmosphere at $37^{\circ} \mathrm{C}$ with $5 \% \mathrm{CO}_{2}$.

115 After $48 \mathrm{~h}$, the nonadherent cells were washed out, and the cultures were expanded to passage 2. 


\section{Co-culture design}

After passage 2, cells were plated in both mono- and co-culture (Fig. 1). In mono-culture

118 experiments, SCs and MSCs were each cultured at $20 \times 10^{3}$ cells per well in a 12 -well gelatin-

119 coated plate, with each condition run in triplicate per animal. In co-culture experiments, SCs and

120 MSCs were each cultured at $20 \times 10^{3}$ cells per well in a 12 -well Transwell ${ }^{\circledR}$ gelatin-coated plate

121 (Corning, Corning, NY), with SCs in the bottom well and MSCs in the insert, separated by the 0.4-

$122 \mu \mathrm{m}$ pore membrane. Plates were allowed to grow to 50\% confluence in SCGM for SCs and in

123 MSCGM for MSCs. At 50\% confluence, media was changed to myogenic media (MM) for SCs

124 (containing high glucose Dulbecco's MEM (Gibco), 1\% horse serum (Gibco), and 100 U/mL

125 penicillin-100 $\mu \mathrm{g} / \mathrm{mL}$ streptomycin (Gibco)) and osteogenic media (OM) for MSCs (containing

126 alpha-MEM (Gibco), 10\% fetal bovine serum (Gibco), $50 \mathrm{mM}$ ascorbic acid (Millipore-Sigma),

$127100 \mu \mathrm{M}$ dexamethasone (Millipore-Sigma), $1 \mathrm{M} \beta$-glycerophosphate (Millipore-Sigma) and 100

$128 \mathrm{U} / \mathrm{mL}$ penicillin-100 $\mu \mathrm{g} / \mathrm{mL}$ streptomycin (Gibco)) to allow differentiation to occur.

129 Differentiation continued for 14 days, at which point outcome metrics (below) were assessed.

\section{Cell proliferation}

132 On differentiation days $0,3,7,10$, and 14 , an alamarBlue ${ }^{\circledR}$ solution (ThermoFisher, Waltham,

133 MA) was mixed into each well $\left(90 \%\right.$ media, $10 \%$ alamarBlue $\left.{ }^{\circledR}\right)$, and the cells were incubated at

$13437^{\circ} \mathrm{C}$. After $3 \mathrm{~h}$, the solution was collected, and absorbance readings were taken at 570 and 600

$135 \mathrm{~nm}$ (Synergy ${ }^{\mathrm{TM}}$ H1M, BioTek Instruments, Inc., Winooski, VT). For the co-cultures, the bottom

136 well and insert of the Transwell $^{\circledR}$ plates were analyzed separately. The percent reduction in

137 alamarBlue ${ }^{\circledR}$ level, a measure of proliferative ability, was determined from the absorbance reading

138 according to the manufacturer's instructions. 


\section{Cell differentiation}

140 On differentiation day 14, cells were rinsed with phosphate buffered saline (PBS), fixed in

141 10\% zinc-buffered formalin (VWR, Randor, PA) for $30 \mathrm{~min}$, and then rinsed again with PBS. To

142 assess mineral deposition for MSC differentiation, a 2\% Alizarin Red S (Fisher) solution was

143 applied to each sample for $5 \mathrm{~min}$, rinsed with deionized water, and imaged using an iPhone 6S

144 camera (Apple, Cupertino, CA) and EVOS ${ }^{\circledR}$ XL light microscope (Life Technologies, Carlsbad,

$145 \mathrm{CA}$ ). Mineral deposition was quantified by solubilizing the Alizarin Red S stain with a $0.5 \mathrm{~N} \mathrm{HCl}$

$146+5 \%$ sodium dodecyl sulfate (SDS) solution for $30 \mathrm{~min}$ and then taking absorbance readings at $147405 \mathrm{~nm}$.

148 To assess SC differentiation, immunocytochemistry (ICC) procedures for skeletal myosin

149 expression were performed. On differentiation day 14, cells were rinsed with DMEM, fixed in $15010 \%$ zinc-buffered formalin for $10 \mathrm{~min}$, and then rinsed with tris-buffered saline (TBS). A blocking

151 solution of TBS with $2 \%$ normal goat serum (NGS) was applied to the cells and allowed to incubate

152 at $4^{\circ} \mathrm{C}$ overnight. Cells were then allowed to warm to room temperature for $10 \mathrm{~min}$. A rat-specific

153 primary antibody for skeletal muscle myosin heavy chain (MA1-35718, ThermoFisher) was

154 diluted 1:5 in TBS-NGS. Wells were rinsed with a TBS-0.05\% Tween 20 (TW20, ThermoFisher)

155 solution, and primary antibodies were applied. Cells were incubated for $1 \mathrm{~h}$ at room temperature,

156 followed by an overnight incubation at $4{ }^{\circ} \mathrm{C}$ in a humidified chamber with gentle shaking. Cells

157 were again allowed to return to room temperature while the secondary antibody (AlexaFluor 584, 158 A-21044, ThermoFisher) was diluted 1:10 in TBS-NGS. Cells were rinsed in TBS-TW20, and the 159 secondary antibody was applied for $2 \mathrm{~h}$ at room temperature. Wells were aspirated and rinsed with 160 TBS-TW20, and a diluted DAPI (ThermoFisher) solution was prepared $(1 \mathrm{ug} / \mathrm{mL}$ diluted in TGS161 NGS) and applied to the wells for $30 \mathrm{~min}$ at room temperature. Wells were rinsed with TBS-TW20 
162 a final time and mounted with a drop of Vectashield (Vector Laboratories, Burlingame, CA) and

163 a $25 \%$ glycerol solution in TBS. Cells were imaged (EVOS ${ }^{\circledR}$ FL Auto, Life Technologies,

164 Carlsbad, CA), and area and integrated density of the fluorescent regions were measured. SC and

165 MSC differentiation were also assessed qualitatively based on cell morphology, looking for multi-

166 nucleated and elongated cells with SCs and a fibroblastic appearance and mineral nodules with

167 MSCs.

168

169

\section{Cellular crosstalk}

170 Since IGF-1 aids growth in both bone and muscle, its expression was assessed in the 171 conditioned media of both mono- and co-cultures at differentiation day 14 using an ELISA kit 172 (ERIGF1, ThermoFisher), according to the manufacturer's instructions. A standard curve was 173 produced, and samples expressing IGF-1 levels outside of the standards' range were omitted.

174 To assess the effect of aging on collagen production, ICC was performed to evaluate the 175 amount of collagen I deposited by the cells. The ICC protocol was the same as for the cellular 176 differentiation analysis, except with a rat-specific collagen I primary antibody (PA1-36145,

177 ThermoFisher) and a secondary antibody (A-11034, ThermoFisher) used at a 1:10 dilution in TBS178 NGS.

181 Statistical analyses were performed with Prism (version 6.07; GraphPad Software, La Jolla,

182 CA) using a significance level of 0.05 . All results were averaged across the groups and expressed

183 in the form of mean \pm standard error of the mean due to unbalanced samples. The effects of age

184 (young, old) and cell type (Mono-SC, Mono-MSC, Co-SC, Co-MSC) were examined using two- 
way ANOVAs. In cases where differentiation day was considered, a two-way ANOVA was performed with repeated measures. Post-hoc pairwise comparisons within each factor were

187 evaluated with Tukey tests if interactions were previously found to be significant.

\section{Results}

\section{Cell viability}

Percent reduction in alamarBlue ${ }^{\circledR}$ differed significantly by cell culture type at differentiation

192 days 7,10 , and 14 ( $\mathrm{p}<0.05$, Fig. 2), with mono-cultures demonstrating greater proliferation than

193 co-cultures at these later time points, and with co-cultures demonstrating no difference between

194 young and old cell types. Young muscle (SCs) in mono-culture demonstrated greater proliferation

195 at days 7,10 and 14 of differentiation compared to young muscle paired with either young or old

196 bone (MSCs) cells ( $\mathrm{p}<0.05$, Fig. 2A). This pattern continued in young bone (Fig. 2B), old muscle

197 (Fig. 2C) and old bone (Fig. 2D). Furthermore, no differences were found in proliferation between

198 young and old. Young monocultures were similar to old monocultures in proliferation, as well as

199 co-cultures. Additionally, there were no differences between young bone and old bone, or young

200 muscle and old muscle.

\section{Collagen and Myosin Content}

203 Collagen immunostaining did not differ across cell or culture type (Fig. 3A). In general,

204 differences in collagen expression between mono-cultures and co-cultures were small $(\mathrm{p}>0.05)$,

205 according to the amount of collagen staining in a given field. When these values were normalized

206 to intensity or number of cells in the area, there still was no difference. For myosin, the relative

207 expression in muscle cells compared to that in bone cells was generally greater in co-culture than 
208 in mono-culture $(\mathrm{p}<0.05$, Fig. 3B), with no difference in the amount of myosin expressed between

209 young and old cells, either in mono- or co-culture $(\mathrm{p}>0.05)$. In mono-culture, myosin expression

210 did not differ between muscle and bone cells regardless of age $(p>0.05)$. However, myosin

211 expression in muscle cells seemed to trend higher than in bone cells. In co-culture, bone cells

212 generally had little to no expression of myosin relative to their muscle cell counter-parts $(\mathrm{p}<0.05)$.

213 Age had no effect on the amount of myosin expressed in these co-cultures. Similarly, when

214 controlling for intensity or number of cells within the field, these differences remained the same

215 (data not shown). Representative images of these condition are shown in Figures 3 C, D, and E.

\section{Mineralization results}

218 Alizarin red results in mono-culture showed that bone cells had or tended to have 219 significantly more amounts of mineralization relative to muscle cells $(\mathrm{p}<0.05)$, yet age did not

220 have a significant effect ( $p>0.05$, Fig. 4A). In co-culture, however, all cell types and ages had 221 very little mineralization $(\mathrm{p}>0.05)$. Between mono- and co-cultures, mineralization was higher in 222 bone cells in mono-culture than in co-culture. These data suggests that osteoblasts more 223 successfully differentiated into osteoblasts in mono-culture than in co-culture.

\section{IGF-1 Content}

ELISA results for IGF-1 expression in conditioned media demonstrated no difference

227 between cell or culture type (Fig. 5). In mono-culture, bone cells tended to have lower expression 228 of IGF-1 compared to muscle cells, but these differences were not significant; young and old cells

229 had similar IGF-1 expression in mono-culture. In co-culture, IGF-1 expression did not differ 230 between cell types or between young and old cells. Furthermore, no differences were found in the 
231 amount of expression between mono- and co-cultures. These results suggest that IGF-1 expression

232 is not altered by communication between these two cell types in aging.

\section{Discussion} understand musculoskeletal diseases. Understanding these interactions with regards to aging could

237 lead to novel therapeutic options to improve aging outcomes. Here, we utilized a previously 238 optimized co-culture system to investigate the bone-muscle interactions in both young and old

239 cells. Our hypothesis was that young cells would generally demonstrate greater proliferation and

240 differentiation compared to old cells. Furthermore, when placed in co-culture, young cells paired

241 together would experience increased crosstalk between the cell types; young cells paired with old 242 cells would be able to improve the old cells' proliferation, differentiation, and crosstalk; and old

243 cells paired together would perform the worst in regard to proliferation, differentiation, and 244 crosstalk. The results of these experiments demonstrated that our hypotheses were partially 245 supported.

\section{Cellular proliferation}

$248 \quad$ Our primary hypotheses for cellular proliferation predicted that young cells would proliferate 249 more than old cells, and cells in co-culture would synergistically undergo greater proliferation 250 compared to the mono-cultured cells. Neither of these hypotheses were supported by the 251 alamarBlue ${ }^{\circledR}$ results, which showed no difference in proliferation between young and old cells and 252 greater proliferative ability at later days of differentiation for mono-cultures compared to co-

253 cultures. Other studies investigating the proliferative potential between two cell types have 
254 typically demonstrated a greater proliferation response for co-cultures compared to mono-

255 cultures. $^{23,24}$ The differences between these finding and our own, however, lie in the selection of

256 animal model, cell type, and type of co-culture. In our case, we are the first to use this animal

257 model of aging in a co-culture study between SCs and MSCs. The decreased proliferation found

258 in our co-cultures may be the result of a regulatory effect between the two cell types in co-culture.

259 This response may mimic those found in the in vivo state, where bone-muscle interactions can

260 restrict the growth of both tissue types. ${ }^{25}$ More interestingly, however, is that this restrict effect

261 was conserved across age in our data. Aging can hinder many aspects of the cellular lifecycle, such

262 as a characteristically reduced proliferative capacity. ${ }^{26-28}$ Our old cell data did not demonstrate this

263 reduced proliferation compared to young cells and may indicate a less severe aging phenotype in

264 our cells than present in other studies. This less severe phenotype was observed not only in

265 proliferation but many of the cellular activities in our data set.

\section{Cellular differentiation}

For differentiation, our hypotheses were 1) old SCs would have a lower capacity to

269 differentiate (measured by skeletal myosin deposition) compared to young SCs; 2) SCs overall

270 would have larger amounts of myosin compared to MSCs; 3) co-cultured SCs would

271 synergistically, due to the presence of MSCs, have more myosin deposition compared to mono-

272 cultured SCs; and 4) the presence of young MSCs would induce old SCs to produce greater

273 amounts of myosin compared to old SCs cultured with old MSCs. Here, our hypotheses were only

274 partially supported. In co-culture particularly, SCs had greater myosin content compared to MSCs,

275 yet age had no significant effect. Other studies have shown that the amount of myosin production

276 decreases in old SCs as they become exhausted, less proliferative, and shift to producing more 
277 collagen or other fibrotic material instead. ${ }^{29,30}$ In mono-culture, myosin expression was not 278 significantly different between SCs and MSCs, although SC myosin expression tended to be 279 greater. These data suggest that, in co-culture, MSCs may have a beneficial effect on the 280 differentiation of SCs. Another study utilizing co-culture in other cell types (e.g., MSCs and 281 cardiomyocytes) also demonstrated this synergistic response, suggesting that crosstalk signals 282 between two cell types may be the source of enhanced differentiation. ${ }^{31}$ Taken together with the 283 viability results, this suggests that instead of actively dividing, the SCs in co-culture conditions 284 favored differentiation more so than in mono-culture. In the future, a more morphological 285 investigation of myoblast fusion could provide a more definitive explanation for differentiation 286 changes as described by others previously. Morphological images taken at time points throughout 287 differentiation could demonstrate shifts in the timing of myoblast fusion with age. demonstrated greater mineral deposition, indicative of increased differentiation, in mono-cultures 290 compared to co-cultures, with little effect of age. We hypothesized that MSCs would have more 291 mineralization in co-culture, with greater effects in young cells, neither of which were supported 292 by our data. We posit two explanations for these observations: 1) SCs inhibited MSC 293 differentiation or 2) media mixing between the two wells diluted differentiation media in MSC 294 wells or a combination of the two. In the first case, experiments exploring bone-muscle crosstalk 295 using isolated monocultures and conditioned media have demonstrated that signaling factors from 296 muscle, such as myostatin and ciliary neurotrophic factor 1 (CNF-1), inhibit osteoblast 297 differentiation. ${ }^{32,33}$ These factors are secreted by muscle after sufficient growth occurs to inhibit 298 further muscle growth. In our preliminary studies and those of others, SCs have been observed to 299 reach myoblast fusion as early as 10 days, ${ }^{34-36}$ whereas MSCs differentiation can take as long as 
300

301

302

303

304

305

306

307

308

309

310

311

312

313

314

315

21 days in some studies ${ }^{37,38}$. We suggest that SCs were reaching a more differentiated state more quickly than MSCs and may have secreted these inhibitory factors before MSCs could completely differentiate. We also found that growth of myoblasts occurred more quickly than MSCs and would result in monolayers lifting off the plate after 14 days, precluding us from continuing to 21 days to see if MSCs could reach a more differentiated state. In the second case, the Transwell ${ }^{\circledR}$ setup required that each cell type receive its own differentiation media, yet the pores within the Transwell $^{\circledR}$ did not inhibit mixing between the two wells. While another study reported that this mixing can take several days ${ }^{39}$, and may not have been a factor in this study, we cannot exclude this possibility. In either case, the presence of significant mineralization in mono-cultures suggest that that the differences have arisen due to the co-culture setup itself. Furthermore, the lack of difference between young and old cultures continues to reinforce that the cells developed a less severe aging phenotype.

\section{Cellular crosstalk}

To investigate crosstalk, we investigated the role of IGF-1 in signaling between the two cell types, as well as collagen I expression in the two cell types. With age, muscle commonly loses its ability to repair and replace tissue effectively and instead deposits collagen. Additionally, bone experiences collagen loss, resulting in an increased incidence of fractures and microcracks with age. ${ }^{9}$ IGF-1 is a potent growth factor of both muscle and bone and can enhance collagen production. We hypothesized that, with age, IGF-1 expression would be decreased, leading to decreases in collagen I deposition in both SCs and MSCs. In co-culture experiments, we hypothesized that old cells plated with young cells would behave more similarly to young cells and have similar IGF-1 and collagen I expression, but our results did not support these hypotheses. 
323 Instead, our results show that IGF-1 expression (Fig. 5) and collagen expression (Fig. 3A) did not

324 differ across the culture conditions, cell types, or age. Many studies have highlighted the role of

325 crosstalk between muscle and bone and the importance of this crosstalk for the growth and

326 development of both. ${ }^{5}$ In this study, we focused on IGF-1, as it is an important mediator of growth

327 and is often reduced with age. ${ }^{40}$ However, IGF-1 has only been minimally investigated during

328 bone-muscle crosstalk.

329 Our results with no age-related changes in IGF-1 expression were surprising, considering the

330 differences in IGF-1 expression observed in several aging studies, but this discrepancy may result

331 from the differences in study design such as using in vitro experiments instead of in vivo

332 experiments. IGF-1 expression is frequently measured in serum samples ${ }^{41}$ or in whole tissue

333 samples. ${ }^{42}$ While both bone and muscle can secrete and react to their own IGF-1 signals in an

334 autocrine fashion, perhaps the IGF-1 expression in bone and muscle in an in vivo environment are

335 enhanced by additional IGF-1 from the bloodstream and elsewhere. In the isolated in vitro

336 environment used here, we found that IGF-1 is clearly secreted by bone and muscle but may only

337 promote initial cell growth and not the continued growth and differentiation. Therefore, while we

338 did not observe differences in IGF-1 expression associated with age or co-culture conditions in

339 this isolated system, it cannot capture the system-wide IGF-1 signaling that occurs in vivo.

340 Furthermore, IGF-1 is merely one bone-muscle crosstalk factor that can be detrimentally affected

341 by aging; studies of additional signaling factors could provide a more complete picture of age-

342 related crosstalk changes in vitro. ${ }^{43-45}$

343 IGF-1 was also investigated due to its significant role in downstream collagen production.

344 We anticipated diminished amounts of IGF-1 expression with aging would lead to decreased

345 collagen deposition in old cells compared with young cells. In this context, the lack of differences 
346 in collagen I expression between cell types, ages, or culture types may not be so surprising. In

347 typical in vivo environments, bone has approximately $36 \%$ of its composition as collagen I, and

348 this content decreases with age. ${ }^{46,47}$ Conversely, muscle has 2-6\% collagen I, and content increases

349 with age. ${ }^{48,49}$ However, collagen I deposition in vitro was not different between bone and muscle

350 cells. Since collagen is deposited by mature cells, and given the early maturation stage of our cells,

351 perhaps the differentiation time was not sufficient to allow significant collagen deposition.

352 Furthermore, in co-culture, the lack of difference in collagen deposition may be indicative of a

353 lack of influence of the crosstalk between the two cell types. While differences in IGF-1 expression

354 or collagen deposition were not observed with co-culture, we cannot exclude the possibility that

355 other signaling pathways or protein expression were not altered by crosstalk. Further studies are

356 required to probe this possibility.

\section{Conclusions}

In summary, we demonstrated that co-culture of bone and muscle cells regulates proliferation

more so than for cells grown in mono-culture, yet age or cell type did not affect proliferation.

361 Furthermore, differences in cellular differentiation or crosstalk were minimal, with little to no

362 effect of age, cell type, or culture condition on myosin expression, mineralization, IGF-1

363 expression, or collagen deposition. This study was the first to use the F344 x BN F1 hybrid rat in

364 a study for cellular aging while also investigating the crosstalk between SCs and MSCs. The lack

365 of differences between young and old cellular proliferation, differentiation, and crosstalk

366 demonstrate that this particular model may not be ideal for age-related cellular studies. However,

367 the use of this model to successfully grow SCs and MSCs should provide evidence to continue

368 using this system for in vitro aging crosstalk studies. The use of a Transwell ${ }^{\circledR}$ co-culture system 
369 provides a framework with which to understand the individual contributions of each cell type,

370 which cannot be isolated in direct co-culture models. Many potential bone-muscle crosstalk factors

371 besides IGF-1 could be contributing to the concomitant age-related changes observed in bone and

372 muscle in vivo, and future studies should focus either on developing in vitro systems that better

373 mimic the in vivo aging environment and/or examining contributions of different signaling

374 pathways to tissue aging. Overall, this study demonstrated that the intricate interactions between

375 bone and muscle depends on a variety of cues, and advancing our understanding of these cues is

376 essential for developing better models for mimicking aging and better treatments to mitigate

377 functional musculoskeletal deficits with aging. 


\section{References}

379 1. Lockhart TE, Smith JL, Woldstad JC. Effects of aging on the biomechanics of slips and falls. Hum Factors. 2005;47(4):708-729. doi: 10.1518/001872005775571014 [doi].

2. Fricke O, Schoenau E. The 'functional muscle-bone unit': Probing the relevance of mechanical signals for bone development in children and adolescents. Growth Horm IGF Res.

3. Freemont AJ, Hoyland JA. Morphology, mechanisms and pathology of musculoskeletal ageing. J Pathol. 2007;211(2):252-259. doi: 10.1002/path.2097 [doi].

4. Marcell TJ. Sarcopenia: Causes, consequences, and preventions. J Gerontol A Biol Sci Med Sci. 2003;58(10):M911-6.

5. Brotto M, Bonewald L. Bone and muscle: Interactions beyond mechanical. Bone. 2015;80:109-

$$
\text { 114. doi: 10.1016/j.bone.2015.02.010 [doi]. }
$$

6. Brotto M, Johnson ML. Endocrine crosstalk between muscle and bone. Curr Osteoporos Rep.

7. Brack AS, Conboy MJ, Roy S, et al. Increased wnt signaling during aging alters muscle stem cell fate and increases fibrosis. Science. 2007;317(5839):807-810. doi: 317/5839/807 [pii].

8. Yin H, Price F, Rudnicki MA. Satellite cells and the muscle stem cell niche. Physiol Rev. 2013;93(1):23-67. doi: 10.1152/physrev.00043.2011 [doi].

9. Boskey AL, Coleman R. Aging and bone. J Dent Res. 2010;89(12):1333-1348. doi: 10.1177/0022034510377791 [doi].

10. Bikle DD, Tahimic C, Chang W, Wang Y, Philippou A, Barton ER. Role of IGF-I signaling in muscle bone interactions. Bone. 2015;80:79-88. doi: 10.1016/j.bone.2015.04.036 [doi]. 
regulating osteoclastogenesis. $J$ Bone Miner Res. 2006;21(9):1350-1358. doi:

12. Jaynes JB, Johnson JE, Buskin JN, Gartside CL, Hauschka SD. The muscle creatine kinase gene is regulated by multiple upstream elements, including a muscle-specific enhancer. Mol Cell Biol. 1988;8(1):62-70.

13. Wang Y, Cheng Z, Elalieh HZ, et al. IGF-1R signaling in chondrocytes modulates growth plate development by interacting with the PTHrP/ihh pathway. J Bone Miner Res. 2011;26(7):14371446. doi: 10.1002/jbmr.359 [doi].

14. Atti E, Boskey AL, Canalis E. Overexpression of IGF-binding protein 5 alters mineral and matrix properties in mouse femora: An infrared imaging study. Calcif Tissue Int. 2005;76(3):187-193. doi: 10.1007/s00223-004-0076-2 [doi].

15. Barton ER, Morris L, Musaro A, Rosenthal N, Sweeney HL. Muscle-specific expression of insulin-like growth factor I counters muscle decline in mdx mice. J Cell Biol. 2002;157(1):137148. doi: $10.1083 /$ jcb.200108071 [doi].

16. Velloso CP. Regulation of muscle mass by growth hormone and IGF-I. Br J Pharmacol. 2008;154(3):557-568. doi: 10.1038/bjp.2008.153 [doi].

17. Conboy MJ, Conboy IM, Rando TA. Heterochronic parabiosis: Historical perspective and methodological considerations for studies of aging and longevity. Aging Cell. 2013;12(3):525530. doi: 10.1111/acel.12065 [doi].

18. Conboy IM, Conboy MJ, Wagers AJ, Girma ER, Weissman IL, Rando TA. Rejuvenation of aged progenitor cells by exposure to a young systemic environment. Nature. 2005;433(7027):760-764. doi: nature03260 [pii].

19. Fleming ME, Watson JT, Gaines RJ, O'Toole RV, Extremity War Injuries VII Reconstruction 
Panel. Evolution of orthopaedic reconstructive care. J Am Acad Orthop Surg. 2012;20 Suppl 1:S74-9. doi: 10.5435/JAAOS-20-08-S74 [doi].

20. Harry LE, Sandison A, Paleolog EM, Hansen U, Pearse MF, Nanchahal J. Comparison of the healing of open tibial fractures covered with either muscle or fasciocutaneous tissue in a

21. Zhang L, Chan C. Isolation and enrichment of rat mesenchymal stem cells (MSCs) and murine model. J Orthop Res. 2008;26(9):1238-1244. doi: 10.1002/jor.20649 [doi]. separation of single-colony derived MSCs. $J$ Vis Exp. 2010;(37). pii: 1852. doi(37):10.3791/1852. doi: 10.3791/1852 [doi].

22. Danoviz ME, Yablonka-Reuveni Z. Skeletal muscle satellite cells: Background and methods

23. Steward AJ, Cole JH, Ligler FS, Loboa EG. Mechanical and vascular cues synergistically for isolation and analysis in a primary culture system. Methods Mol Biol. 2012;798:21-52. doi:

24. Walenda T, Bork S, Horn P, et al. Co-culture with mesenchymal stromal cells increases proliferation and maintenance of haematopoietic progenitor cells. J Cell Mol Med. 2010;14(12):337-350. doi: 10.1111/j.1582-4934.2009.00776.x [doi].

444 26. Li Y, Charif N, Mainard D, Bensoussan D, Stoltz JF, de Isla N. Donor's age dependent

25. Maurel DB, Jahn K, Lara-Castillo N. Muscle-bone crosstalk: Emerging opportunities for novel proliferation decrease of human bone marrow mesenchymal stem cells is linked to diminished clonogenicity. Biomed Mater Eng. 2014;24(1 Suppl):47-52. doi: 10.3233/BME-140973 [doi]. 
447

448

449

450

451

452

453

454

455

456

457

458

459

460

461

462

463

464

465

466

467

468

469

27. Tsai WC, Chang HN, Yu TY, et al. Decreased proliferation of aging tenocytes is associated with down-regulation of cellular senescence-inhibited gene and up-regulation of $\mathrm{p} 27$. J Orthop Res. 2011;29(10):1598-1603. doi: 10.1002/jor.21418 [doi].

28. Harthan LB, McFarland DC, Velleman SG. Changes in proliferation, differentiation, fibroblast growth factor 2 responsiveness and expression of syndecan-4 and glypican-1 with turkey satellite cell age. Dev Growth Differ. 2013;55(5):622-634. doi: 10.1111/dgd.12069 [doi].

29. Thompson LV. Age-related muscle dysfunction. Exp Gerontol. 2009;44(1-2):106-111. doi: 10.1016/j.exger.2008.05.003 [doi].

30. Miljkovic N, Lim JY, Miljkovic I, Frontera WR. Aging of skeletal muscle fibers. Ann Rehabil Med. 2015;39(2):155-162. doi: 10.5535/arm.2015.39.2.155 [doi].

31. Plotnikov EY, Khryapenkova TG, Vasileva AK, et al. Cell-to-cell cross-talk between mesenchymal stem cells and cardiomyocytes in co-culture. $J$ Cell Mol Med. 2008;12(5A):1622-1631. doi: JCMM205 [pii].

32. Johnson RW, White JD, Walker EC, Martin TJ, Sims NA. Myokines (muscle-derived cytokines and chemokines) including ciliary neurotrophic factor (CNTF) inhibit osteoblast differentiation. Bone. 2014;64:47-56. doi: 10.1016/j.bone.2014.03.053 [doi].

33. Qin Y, Peng Y, Zhao W, et al. Myostatin inhibits osteoblastic differentiation by suppressing osteocyte-derived exosomal microRNA-218: A novel mechanism in muscle-bone communication. J Biol Chem. 2017;292(26):11021-11033. doi: 10.1074/jbc.M116.770941 [doi].

34. Schuierer MM, Mann CJ, Bildsoe H, Huxley C, Hughes SM. Analyses of the differentiation potential of satellite cells from myoD-/-, mdx, and PMP22 C22 mice. BMC Musculoskelet Disord. 2005;6:15-2474-6-15. doi: 1471-2474-6-15 [pii]. 
35. O'Connor MS, Carlson ME, Conboy IM. Differentiation rather than aging of muscle stem cells abolishes their telomerase activity. Biotechnol Prog. 2009;25(4):1130-1137. doi: 10.1002/btpr.223 [doi].

36. Gabillard JC, Sabin N, Paboeuf G. In vitro characterization of proliferation and differentiation of trout satellite cells. Cell Tissue Res. 2010;342(3):471-477. doi: 10.1007/s00441-010-1071-

37. Hanna H, Mir LM, Andre FM. In vitro osteoblastic differentiation of mesenchymal stem cells generates cell layers with distinct properties. Stem Cell Res Ther. 2018;9(1):203-018-0942-x.

38. Zhang W, Yang N, Shi XM. Regulation of mesenchymal stem cell osteogenic differentiation by glucocorticoid-induced leucine zipper (GILZ). J Biol Chem. 2008;283(8):4723-4729. doi: M704147200 [pii].

39. Renaud J, Martinoli MG. Development of an insert co-culture system of two cellular types in

40. Balasubramanian P, Longo VD. Growth factors, aging and age-related diseases. Growth Horm IGF Res. 2016;28:66-68. doi: 10.1016/j.ghir.2016.01.001 [doi].

41. Gong Z, Kennedy O, Sun H, et al. Reductions in serum IGF-1 during aging impair health span. Aging Cell. 2014;13(3):408-418. doi: 10.1111/acel.12188 [doi]. and longevity. Nat Rev Endocrinol. 2013;9(6):366-376. doi: 10.1038/nrendo.2013.67 [doi]. 
493 44. Novotny SA, Warren GL, Hamrick MW. Aging and the muscle-bone relationship. Physiology

494 (Bethesda). 2015;30(1):8-16. doi: 10.1152/physiol.00033.2014 [doi].

495 45. Hamrick MW. The skeletal muscle secretome: An emerging player in muscle-bone crosstalk.

496 Bonekey Rep. 2012;1:60. doi: 10.1038/bonekey.2012.60 [doi].

497 46. Nyman JS, Roy A, Acuna RL, et al. Age-related effect on the concentration of collagen

498 crosslinks in human osteonal and interstitial bone tissue. Bone. 2006;39(6):1210-1217. doi:

499 S8756-3282(06)00580-1 [pii].

500 47. Young MF. Bone matrix proteins: Their function, regulation, and relationship to osteoporosis.

501 Osteoporos Int. 2003;14 Suppl 3:S35-42. doi: 10.1007/s00198-002-1342-7 [doi].

502 48. Gillies AR, Lieber RL. Structure and function of the skeletal muscle extracellular matrix.

503 Muscle Nerve. 2011;44(3):318-331. doi: 10.1002/mus.22094 [doi].

504 49. Lacraz G, Rouleau AJ, Couture V, et al. Increased stiffness in aged skeletal muscle impairs

505 muscle progenitor cell proliferative activity. PLoS One. 2015;10(8):e0136217. doi:

$506 \quad$ 10.1371/journal.pone.0136217 [doi]. 


\section{Figures}

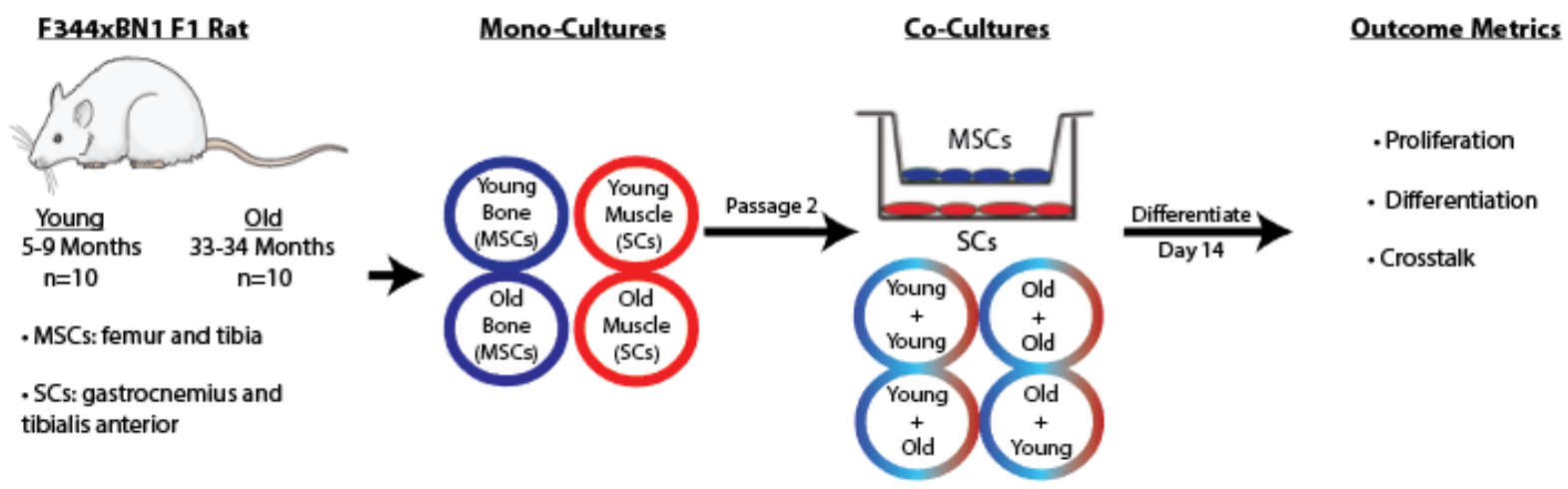

Figure 1. Study design. SCs and MSCs were isolated from rat tissue and cultivated in monoculture and co-culture and allowed to differentiate for 14 days before outcome metrics were assessed. 
A.

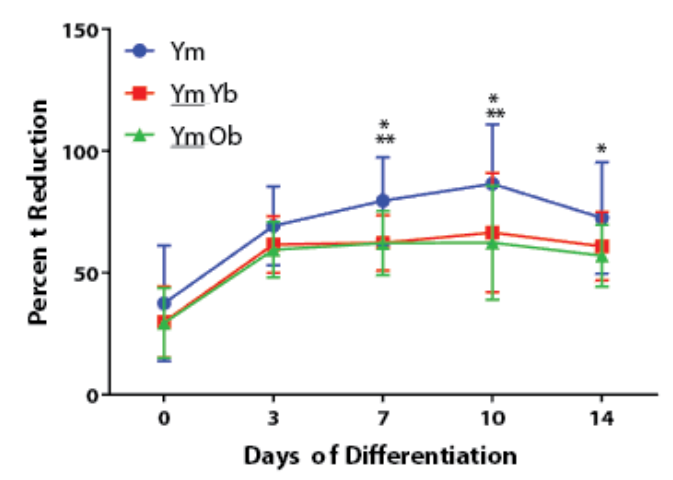

C.

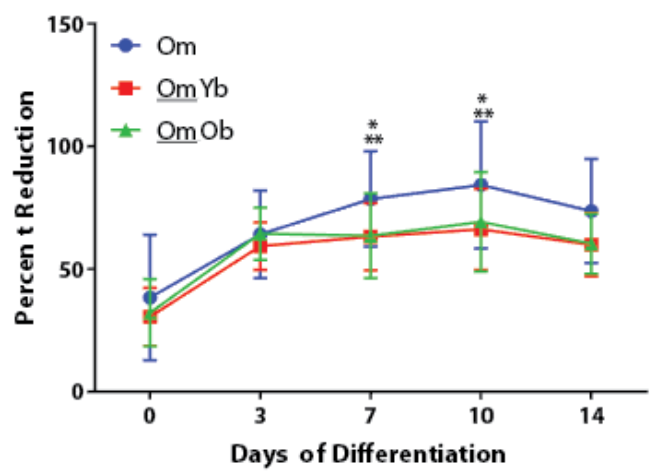

B.

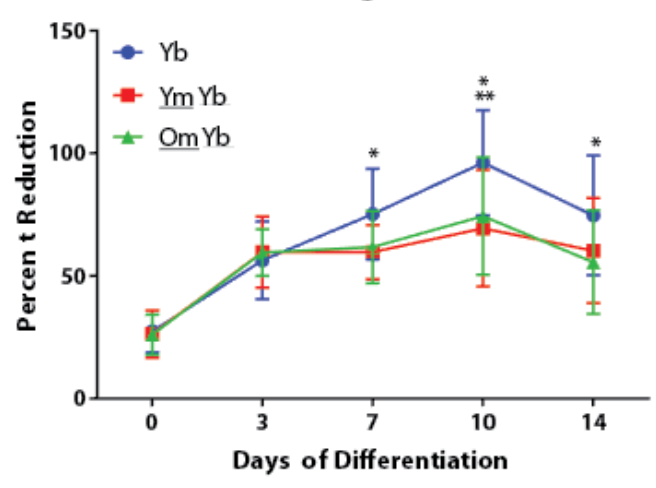

D.

Old Bone

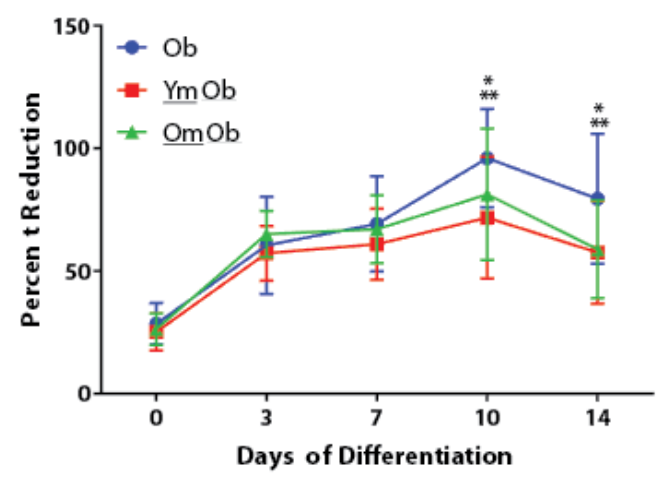

Figure 2. AlamarBlue ${ }^{\circledR}$ reduction across differentiation. Proliferative ability differed between some mono- and co-cultures during differentiation days 7-14 for A) young muscle, B) young bone, C) old muscle, and D) old bone. Data are mean \pm SEM. *p $<0.05$ for mono-culture (blue) vs. coculture (red). ${ }^{* *} \mathrm{p}<0.05$ for mono-culture (blue) vs. co-culture (green). 


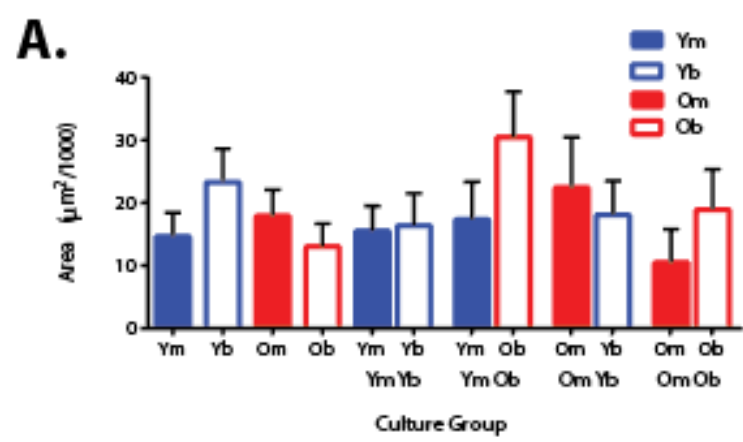

C.

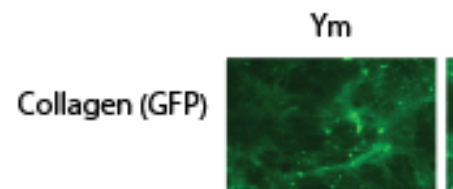

$\mathrm{Yb}$
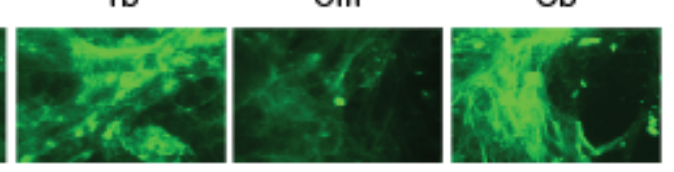

Skeletal Myosin (TxRed)
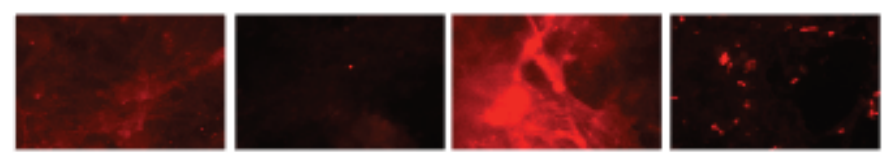

Nuclei (DAPI)
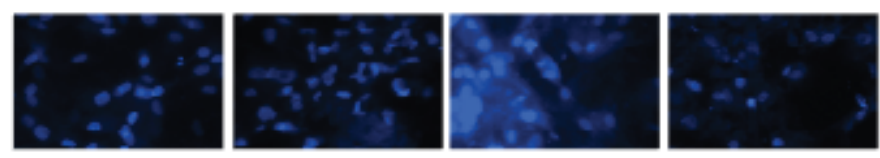

Composite
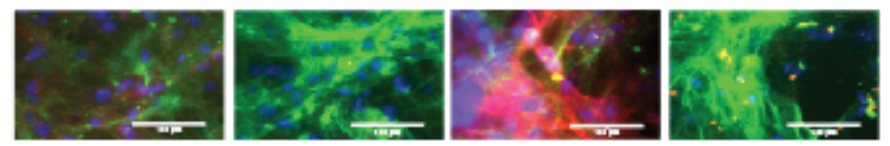

YmOb

D.

Collagen (GFP)

Ym

$\mathrm{Yb}$

Ym

$\mathrm{Ob}$
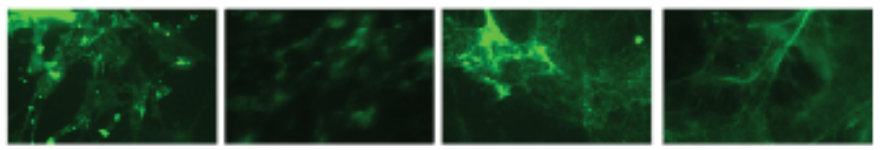

Skeletal Myosin (TxRed)
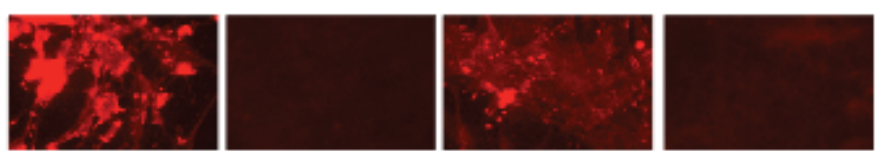

Nuclei (DAPI)
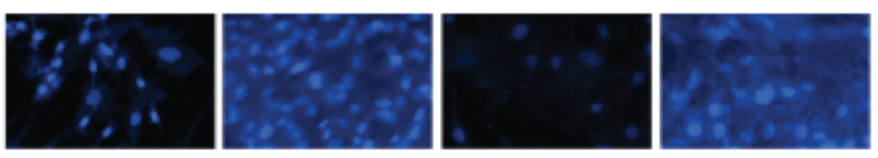

Composite
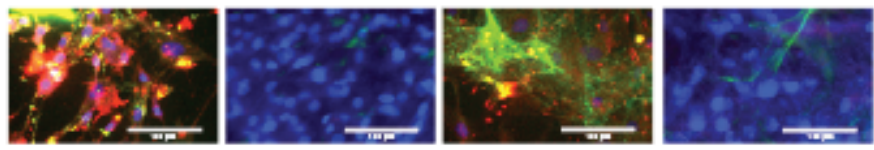
E.

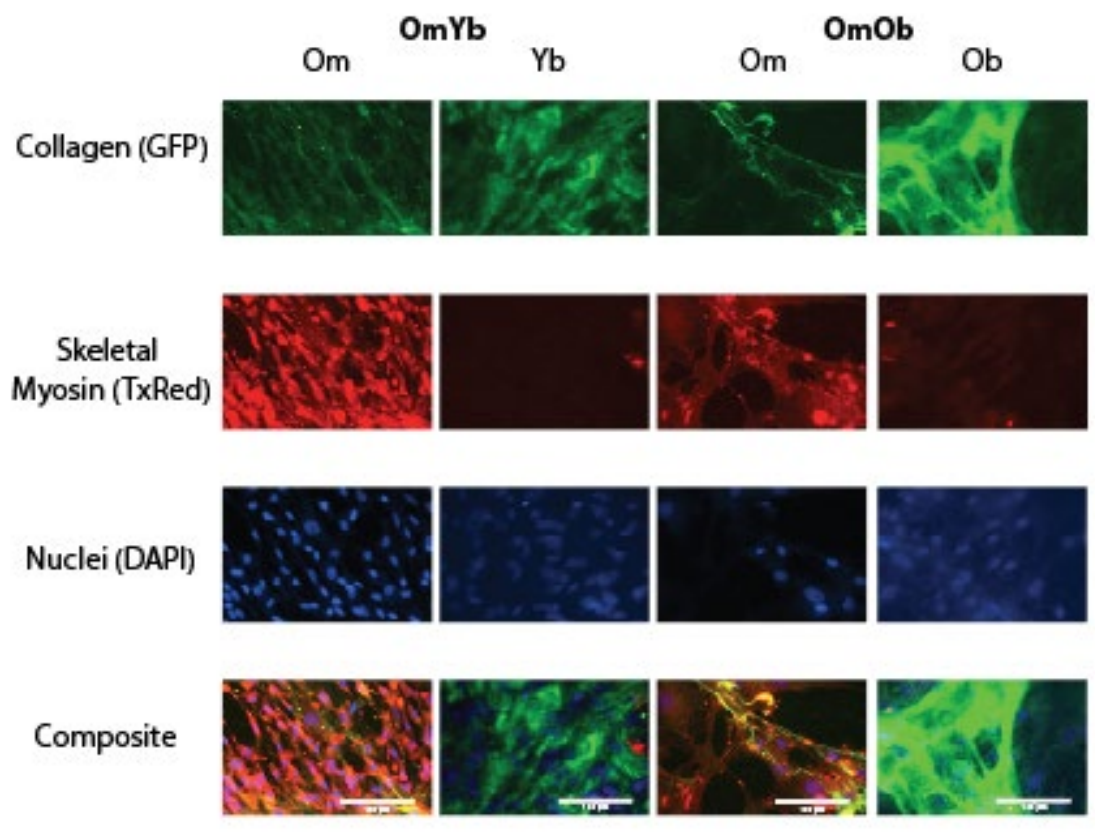

Figure 3. Immunocytochemistry analysis of cultured cells. Collagen I expression (A) and skeletal myosin expression (B) after 14 days of differentiation. No differences in collagen expression were found across cell type or age, or in mono- vs. co-culture $(\mathrm{p}<0.05)$. Myosin expression was typically increased in SCs in co-culture $\left({ }^{*}, p<0.05\right)$ and tended to be increased compared to MSCs in mono-culture. Age had no effect between these conditions. Representative images from the various conditions are shown in (C), (D), and (E). Data are mean $\pm \mathrm{SEM}$. 


\section{A.}

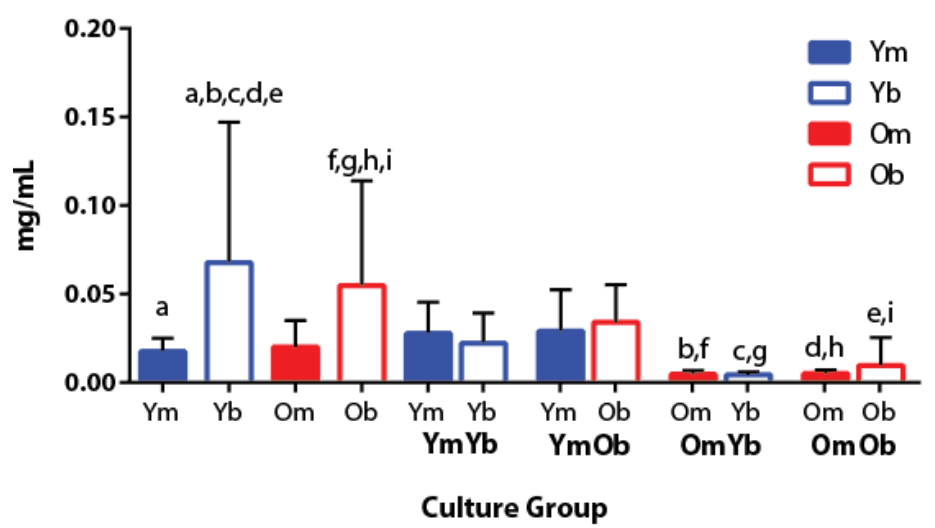

B.

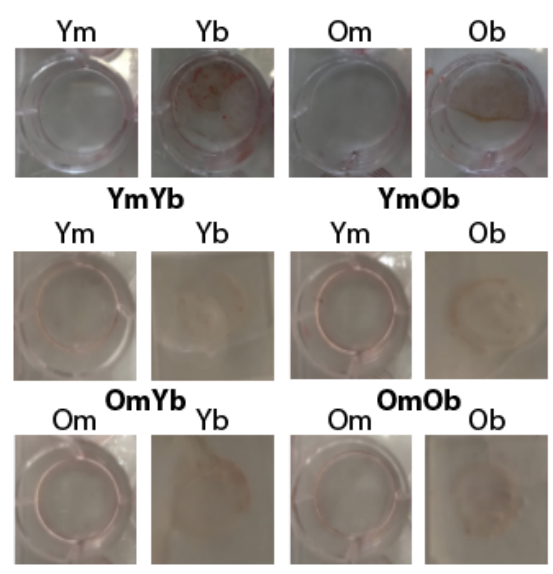

Figure 4. Alizarin red solubilization data from fixed cells. Solubilization results (A) and representative images (B). In mono-culture, MSCs tended to have or had a significantly greater amount of mineralization compared to SCs $(\mathrm{p}<0.05)$. In co-culture, MSCs paired with old SCs had significantly less mineralization compared to their mono-culture equivalent $(\mathrm{p}<0.05)$. MSCs paired with young SCs had similar amounts of mineralization compared to mono-culture $(\mathrm{p}<$ 0.05 ). Data are mean $\pm \mathrm{SD}$. Bars with the same letter above them are significantly different. 


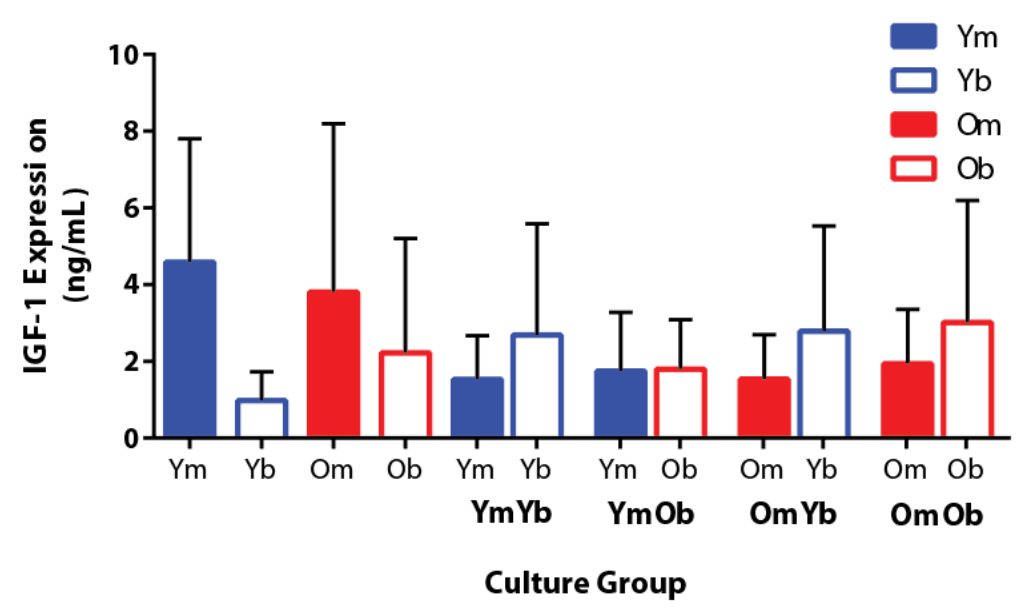

Figure 5. IGF-1 expression from conditioned media on day 14. IGF-1 expression did not differ by cell type, culture type, or age $(p>0.05)$. Data are mean \pm SEM. 